\title{
EEG driven tDCS versus bifrontal tDCS for tinnitus
}

\section{Dirk De Ridder* and Sven Vanneste}

Brai $^{2} n$ and Translational Neuroscience, University Hospital Antwerp, Antwerp, Belgium

Edited by:

Felipe Fregni, Harvard Medical

School, USA

Reviewed by:

Tarek Rajji, Centre for Addiction and Mental Health, Canada

Wolnel Caumo, Universidade Federal do rio Grande do Sul, Brazil

*Correspondence:

Dirk De Ridder, Brai $^{2} n$, University Hospital Antwerp, Wilrijkstraat 10, 2650 Edegem, Antwerp Belgium. e-mail: dirk.de.ridder@uza.be
Tinnitus is the perception of a sound in the absence of any objective physical sound source. Transcranial Direct Current Stimulation (tDCS) induces shifts in membrane resting potentials depending on the polarity of the stimulation: under the anode gamma band activity increases, whereas under the cathode the opposite occurs. Both single and multiple sessions of tDCS over the dorsolateral prefrontal cortex (DLPFC; anode over right DLPFC) yield a transient improvement in tinnitus intensity and tinnitus distress. The question arises whether optimization of the tDCS protocol can be obtained by using EEG driven decisions on where to place anode and cathode. Using gamma band functional connectivity could be superior to gamma band activity as functional connectivity determines the tinnitus network in many aspects of chronic tinnitus. Six-hundred-seventy-five patients were included in the study: 265 patients received tDCS with cathodal electrode placed over the left DLPFC and the anode placed overlying the right DLPFC, 380 patients received tDCS based on EEG connectivity, and 65 received no tDCS (i.e., waiting list control group). Repeated measures ANOVA revealed a significant main effect for pre versus post measurement. Bifrontal tDCS in comparison to EEG driven tDCS had a larger reduction for both tinnitus distress and tinnitus intensity. Whereas the results of the bifrontal tDCS seem to confirm previous studies, the use of gamma band functional connectivity seems not to bring any advantage to tDCS for tinnitus suppression. Using other potential biomarkers, such as gamma band activity, or theta functional connectivity could theoretically be of use. Further studies will have to elucidate whether brain state based tDCS has any advantages over "blind" bifrontal stimulation.

\section{Keywords: tinnitus, EEG, tDCS, direct current, gamma}

\section{INTRODUCTION}

Tinnitus is the perception of a sound or sounds (e.g., a tone, hissing, or buzzing sound, or a combination of such sounds) in the absence of any objective physical sound source (Jastreboff, 1990). In western societies about $5-15 \%$ of the population has chronic tinnitus and will seek medical attention (Axelsson and Ringdahl, 1989; Heller, 2003). Tinnitus often causes a considerable amount of distress: between 6 and $25 \%$ of the affected people report symptoms that are severely debilitating (Baguley, 2002; Eggermont and Roberts, 2004).

Based on functional imaging studies, including fMRI (Smits et al., 2007), EEG (van der Loo et al., 2009; Vanneste et al., 2010a), MEG (Muhlnickel et al., 1998; Weisz et al., 2007), and PET (Lockwood et al., 1999; Langguth et al., 2006) it is generally accepted that tinnitus is related to auditory cortex hyperactivity and maladaptive plasticity, often due to damage of the peripheral auditory system. But co-activation of non-auditory brain structures such as the insula (Smits et al., 2007; Vanneste et al., 2010a; van der Loo et al., 2011), anterior cingulate cortex (Muhlau et al., 2006; Plewnia et al., 2007; Rauschecker et al., 2010; Vanneste et al., 2010a; Leaver et al., 2011; Vanneste et al., 2011c), and dorsolateral prefrontal cortex (DLPFC; Schlee et al., 2009a; Vanneste et al., 2010a) has been described as well, and some pathophysiological mechanisms have been proposed based on these studies (Rauschecker et al., 2010; De Ridder et al., 2011a; Leaver et al., 2011). This has led to the concept that the unified tinnitus percept is the result of one large tinnitus network consisting of multiple dynamically adaptive overlapping subnetworks (De Ridder et al., 2011a), with each subnetwork representing a clinically separable aspect such as distress (Vanneste et al., 2010a; De Ridder et al., 2011b), sound characteristic (noise-like versus pure tone; Vanneste et al., 2010b), lateralization (Vanneste et al., 2011a), etc.

The DLPFC has an important function in auditory processing. Bilateral DLPFC has a facilitatory effect on auditory memory storage and contains auditory memory cells (Bodner et al., 1996). This prefrontal area also exerts early inhibitory modulation of input to primary auditory cortex in humans (Knight et al., 1989) and has been found to be associated with auditory attention (Alain et al., 1998; Lewis et al., 2000; Voisin et al., 2006) resulting in top-down modulation of auditory processing (Mitchell et al., 2005). This has been further confirmed by electrophysiological data indicating that tinnitus might occur as the result of a dysfunction in the topdown inhibitory processes (Norena et al., 1999; Faber et al., 2011).

Transcranial Direct Current Stimulation (tDCS) is an old neuromodulation tool which recently has seen a revival. In tDCS, a weak direct electrical current $(1-2 \mathrm{~mA})$ is applied to the scalp, through which most of the current is shunted. But about $50 \%$ of the transcranially applied direct current reaches the brain, both in animal models (Rush and Driscoll, 1968) and humans (Dymond et al., 1975). This current induces shifts in 
membrane resting potentials, thereby depolarizing or hyperpolarizing neurons (Nitsche et al., 2003) depending on the polarity of the stimulation. tDCS induces an increase or decrease in cortical excitability in the brain regions to which it is applied (Nitsche and Paulus, 2000; Miranda et al., 2006). Anodal tDCS typically has an excitatory effect on the local cortical excitability by inducing a relative neuronal depolarization, while cathode has an opposite effect - it induces a hyperpolarization (Nitsche and Paulus, 2001). tDCS was first applied to the auditory cortex in an attempt to improve tinnitus (Fregni et al., 2006), and does indeed seem to be able to induce long-lasting changes in tinnitus perception (Garin et al., 2011).

Based on the influence of the DLPFC on auditory processing and its involvement in tinnitus it was demonstrated that a single session of tDCS over the DLPFC (anode over right DLPFC) yields a transient improvement in both tinnitus intensity and tinnitus distress in subjects with chronic tinnitus (Vanneste et al., 2010c), where as stimulation with anode over left DLPFC induces no changes in tinnitus (Vanneste et al., 2010c). When applying repetitive sessions this could be proposed as a treatment (Faber et al., 2011; Frank et al., 2012). The efficacy of bifrontal tDCS for transient tinnitus suppression depends on the brain state (Vanneste et al., 2011b). Applying multiple sessions of bifrontal tDCS has been proposed as potential treatment for tinnitus (Frank et al., 2012). The question arises whether optimalization of the tDCS protocol can be obtained by using EEG driven decisions on where to place anode and cathode. Based on the pathophysiology of tinnitus and the polarity dependent effect of tDCS it can be proposed to place the (inhibitory) cathode at an area of tinnitus related gamma band activity (De Ridder et al., 2007, 2011a,c; Lorenz et al., 2009; Schlee et al., 2009b; van der Loo et al., 2009), or even better gamma band functional connectivity (Vanneste et al., 2011b; Schlee et al., 2009a). Using gamma band functional connectivity could be superior to gamma band activity as functional connectivity determines the tinnitus network in many aspects of chronic tinnitus (Vanneste et al., 2010b, 2011b,d; Schlee et al., 2009b; Vanneste and De Ridder, 2011).

\section{METHODS AND MATERIALS PARTICIPANTS}

Six-hundred-seventy-five subjects (260 males and 415 females) with chronic tinnitus ( $>1$ year) were recruited from the Tinnitus Clinic at the University Hospital Antwerp, Belgium and participated in this retrospective study, with a mean age of 48.33 years $(M d=50 ; \mathrm{SD}=14.57)$. The mean tinnitus duration was 5.14 years $(M d=4 ; \mathrm{SD}=4.24)$. In order to obtain a homogeneous sample and exclude potential variables that would interfere with response to tDCS, we excluded subjects based on the following criteria: individuals with pulsatile tinnitus, a history of epileptic insults, severe organic co-morbidity, a pacemaker, or defibrillator, a present pregnancy, neurological disorders such as brain tumors, and individuals being treated for mental disorders. All prospective subjects underwent a complete ENT and neurological investigation to rule out possible treatable causes for their tinnitus. All patients younger than 18 years were excluded from the study. Table 1 further shows the tinnitus characteristics for both groups. The study was in accordance with the ethical standards of the Helsinki declaration (1964)
Table 1 | Tinnitus characteristics.

\begin{tabular}{lllll}
\hline & & \multicolumn{3}{c}{ Groups } \\
\cline { 3 - 5 } & & $\begin{array}{l}\text { Frontal } \\
\text { tDCS }\end{array}$ & $\begin{array}{l}\text { EEG driven } \\
\text { tdcs }\end{array}$ & $\begin{array}{l}\text { Waiting } \\
\text { list }\end{array}$ \\
\hline \multirow{2}{*}{$\begin{array}{l}\text { Mean duration } \\
\text { Type }\end{array}$} & Pure tone & 5.11 & 5.22 & 4.80 \\
\multirow{2}{*}{ Laterality } & 85 & 134 & 21 \\
& Narrow band noise & 188 & 246 & 44 \\
& Unilateral & 148 & 214 & 36 \\
& Bilateral & 117 & 166 & 29 \\
\hline
\end{tabular}

and was approved by the institutional ethics committee of the Antwerp University Hospital.

\section{TRANSCRANIAL DIRECT CURRENT STIMULATION}

Direct current was transmitted by a saline-soaked pair of surface sponges $\left(35 \mathrm{~cm}^{2}\right)$ and delivered by a battery-driven, constant current stimulator with a maximum output of $10 \mathrm{~mA}$ (NeuroConn; http://www.neuroconn.de/). Two hundred sixty-five patients received tDCS with cathodal electrode placed over the left DLPFC and the anode placed overlying the right DLPFC, 380 patients received tDCS based on EEG connectivity. Patients who received $\mathrm{tDCS}$ were randomly assigned to DLPFC $\mathrm{tDCS}$ or $\mathrm{tDCS}$ based on EEG. In addition 65 received no tDCS, and were used as a waiting list control group.

For the EEG driven tDCS, EEGs (Mitsar, Saint Petersburg, Russia) were obtained 1 week before the tACS stimulation in a fully lighted room with each participant sitting upright in a comfortable chair. The EEG was sampled with 19 electrodes (Fp1, Fp2, F7, F3, Fz, F4, F8, T7, C3, Cz, C4, T8, P7, P3, Pz, P4, P8, O1 $\mathrm{O} 2)$ in the standard 10-20 International placements referenced to linked lobes and impedances were checked to remain below $5 \mathrm{k} \Omega$. Data were collected for 1002 -s epochs eyes closed, sampling rate $=1024 \mathrm{~Hz}$, and band passed $0.15-200 \mathrm{~Hz}$. Data were resampled to $128 \mathrm{~Hz}$, band-pass filtered (fast Fourier transform filter) to 2-44 Hz. These data were transposed into Eureka! Software (Congedo, 2002), plotted and carefully inspected for manual artifact rejection. All episodic artifacts including eye blinks, eye movements, teeth clenching, body movement, or ECG artifacts were removed from the stream of the EEG.

\section{TARGET LOCALIZATION}

To determine the precise location of the gamma band functional connectivity, i.e., lagged phase synchronization is used. This was operationally defined as the brain area retrieved on source localized EEG, using sLORETA software, in each individual, which has most connectivity lines in the gamma band $(30-45 \mathrm{~Hz})$. This measure is threshold invariant (when increasing the threshold the amount of functional connections will decrease in all areas, but the area with most connections will remain the area with most functional connections) and clinically applicable. The brain area with the highest gamma band functional connectivity was elected as the target for cathode placement. The area for placing the anode was determined by the highest theta band functional connectivity.

Connectivity can be calculated by analyzing phase synchronization or coherence. However, any measure of dependence is 
highly contaminated with an instantaneous, non-physiological contribution due to volume conduction (Pascual-Marqui, 2007a). Therefore, Pascual-Marqui, (Pascual-Marqui, 2007b) introduced a new technique (i.e., Hermitian covariance matrices) that removes this confounding factor. As such, this measure of dependence can be applied to any number of brain areas jointly, i.e., distributed cortical networks, whose activity can be estimated with sLORETA. Measures of linear dependence (coherence) between the multivariate time series are defined. The measures are expressed as the sum of lagged dependence and instantaneous dependence. The measures are non-negative, and take the value zero only when there is independence and are defined in the gamma $(30.5-45 \mathrm{~Hz})$ frequency domain. Thus only the lagged phase synchronization is used. Regions of interest were defined based on previous brain research on tinnitus (see Table 2 for overview). Based on the functional connectivity analysis the region which forms a hub (i.e., ROI that is connected with the most ROI) is selected as the target area to be stimulated.

\section{EVALUATION}

A visual analog scale for tinnitus intensity ("How loud is your tinnitus?: $0=$ no tinnitus and $10=$ as loud as imaginable") and tinnitus distress ("How stressful is your tinnitus? $0=$ no distress and $10=$ suicidal distress") was asked before (pre) and directly after (post) tDCS stimulation. The responses were collected by the person who applied the tDCS.

\section{STATISTICAL ANALYSES}

A three-stage analysis was performed. First the overall results were calculated to verify whether there was an effect obtained by tDCS in comparison to baseline. This is followed by a second analysis evaluating whether there was a difference between frontal and EEG driven tDCS. This is then followed by a third analysis looking at the response rate and response size differences between bifrontal and EEG driven tDCS. Calculations were performed using SPSS 18.0 software package.

\section{Overall effects}

A repeated measure ANOVA was conducted with VAS distress and VAS intensity pre-tDCS and post tDCS as within-subjects variables and condition (frontal tDCS, EEG driven tDCS, and waiting list) as between-subjects variable. To verify that the within-variables were normally distributed a Kolmogorov-Smirnov test was applied. This demonstrated that the within-variables did not deviate from a normal distribution. In addition we reported the effect size by including the partial eta squared $\left(\eta^{2}\right)$. The standards for these effect sizes are small $\left(\eta^{2}=0.01\right)$, medium $\left(\eta^{2}=0.06\right)$, and large $\left(\eta^{2}=0.14\right)$.

\section{A comparison between the effects obtained for frontal and EEG driven tDCS}

A second repeated measures ANOVA was conducted with the obtained difference (Pre - Post tDCS) as within-subjects variable and group (Frontal tDCS versus EEG driven tDCS) as betweensubjects variable to verify whether there was a significant difference in the obtained suppression on both distress and intensity. In addition we also reported the effect size by including the partial eta squared $\left(\eta^{2}\right)$

\section{The effects for responders only}

We applied a logistic regression with condition (including frontal tDCS and EEG driven tDCS) as independent variable and responding $(\mathrm{No}=0$ or Yes $=1)$ as dependent variable. Responders are defined as patients who obtain minimally $10 \%$ suppression, while non-responders are defined as those patients obtain less than $10 \%$ improvement. A repeated measures ANOVA was conducted with VAS distress and VAS intensity pre-tDCS and post $\mathrm{tDCS}$ as withinsubjects variables and condition (frontal tDCS and EEG driven tDCS) as between group variable to verify if there was a significant difference for the responders only on both distress and intensity. We also reported the effect size by including the partial eta squared $\left(\eta^{2}\right)$.

\section{RESULTS}

\section{OVERALL EFFECTS}

A comparison between the baseline measurements between the three different groups revealed no significant effect for both tinnitus distress $(F=0.24, p=0.79)$ and tinnitus intensity $(F=2.01$, $p=0.13)$.

Table 2 | Regions of interest.

\begin{tabular}{|c|c|c|}
\hline Brodmann area & Brain area & Author \\
\hline BA6 & Supplementary motor area & Jastreboff (1990) \\
\hline BA7 & Precuneus & Heller (2003) \\
\hline BA9-46 & Dorsolateral prefontal cortex & $\begin{array}{l}\text { Heller (2003), Axelsson and Ringdahl (1989), Baguley (2002), Eggermont and Roberts (2004), } \\
\text { Smits et al. (2007), van der Loo et al. (2009) }\end{array}$ \\
\hline BA10 & Frontopolar cortex & Vanneste et al. (2010a), Muhlnickel et al. (1998) \\
\hline BA11 & Orbitofrontal & Vanneste et al. (2010a) \\
\hline BA13 & Insula & Heller (2003), Weisz et al. (2007) \\
\hline BA21-22 & Secondary auditory cortex & Lockwood et al. (1999), Langguth et al. (2006), Muhlnickel et al. (1998) \\
\hline BA23-31 & Posterior cingulate cortex & Heller (2003) \\
\hline BA24-32 & Dorsal anterior cingulate cortex & Heller (2003), Smits et al. (2007) \\
\hline BA25 & Subgenual anterior cingulate cortex & Heller (2003), Smits et al. (2007) \\
\hline BA39-40 & Angular gyrus & Muhlnickel et al. (1998) \\
\hline BA41-42 & Primary auditory cortex & Lockwood et al. (1999), Langguth et al. (2006), Muhlnickel et al. (1998) \\
\hline
\end{tabular}


A repeated measures ANOVA revealed a significant main effect for pre versus post measurement $\left(F=17.19, p<0.001, \eta^{2}=0.05\right)$. A closer look to the data indicated that for distress $(F=25.01$, $\left.p<0.001, \eta^{2}=0.03\right)$ there was a significant decrease in the post tDCS in comparison to pre-tDCS. For intensity a similar effect was obtained $\left(F=31.16, p<0.001, \eta^{2}=0.04\right)$ demonstrating there was a significant decrease in the post tDCS in comparison to pre-tDCS. No significant main effect was obtained for condition (frontal tDCS, EEG driven tDCS, and waiting list) on both tinnitus distress and tinnitus intensity. In addition an interaction effect was obtained between condition (frontal tDCS, EEG driven tDCS, and waiting list) $\times$ tDCS (pre versus post) for both tinnitus distress and tinnitus intensity $\left(F=6.59, p<0.001, \eta^{2}=0.02\right)$. For tinnitus distress it was shown that both frontal tDCS $(F=68.73$, $\left.p<0.001, \eta^{2}=0.07\right)$ and EEG driven tDCS $(F=7.42, p<0.01$, $\left.\eta^{2}=0.04\right)$ had post tDCS a significant reduction in comparison

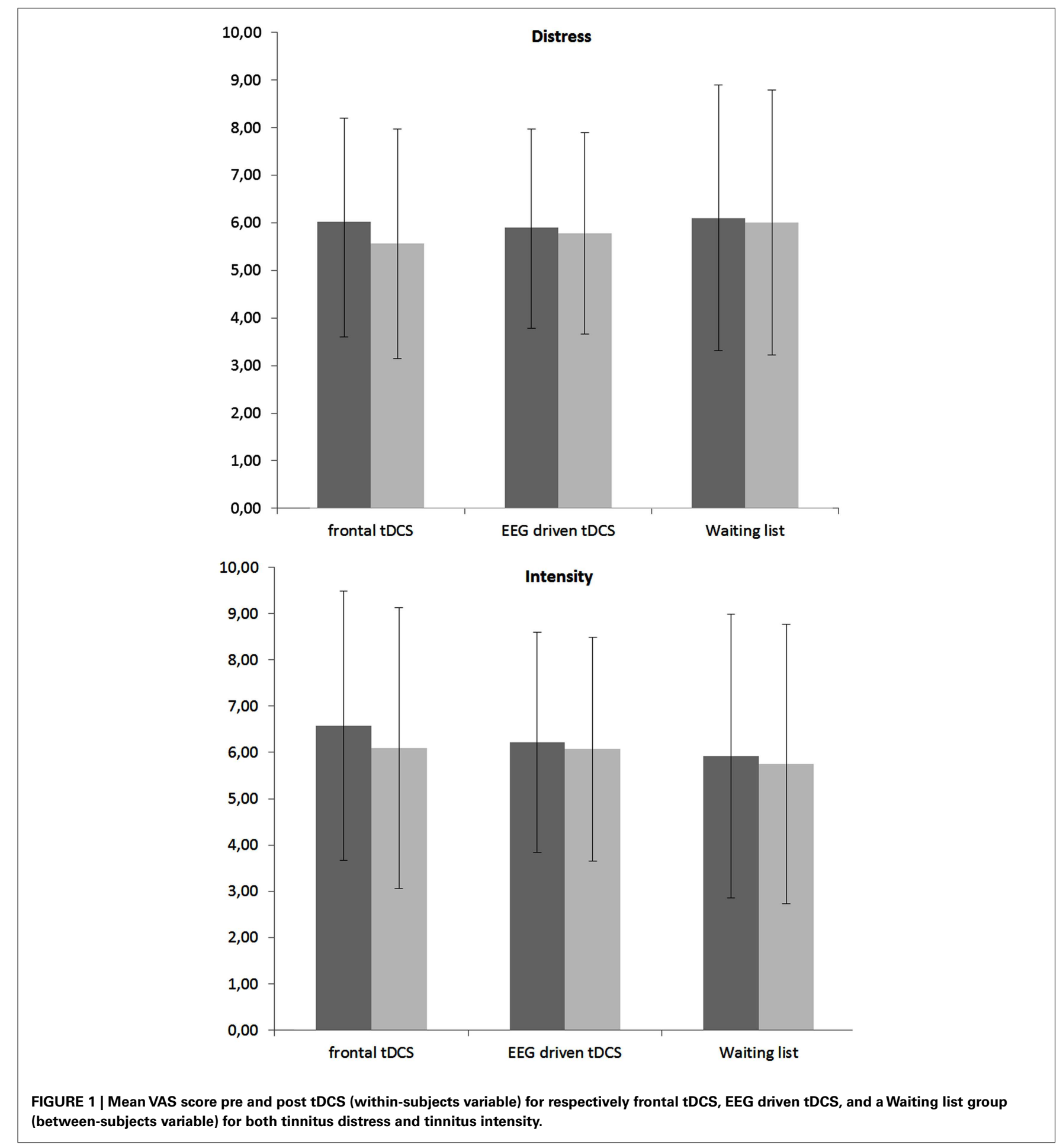


to pre-tDCS scores (see Figure 1). No effect was obtained for the waiting list group. For tinnitus intensity also a significant decrease was demonstrated post tDCS in comparison to pre-tDCS scores for respectively frontal tDCS $\left(F=69.95, p<0.001, \eta^{2}=0.09\right)$ and EEG driven tDCS $\left(F=9.39, p<0.01, \eta^{2}=0.01\right)$. Again, no effect was obtained for the waiting list group. Figure 1 gives an overview of the obtained results.

\section{A COMPARISON BETWEEN THE EFFECTS OBTAINED FOR FRONTAL AND EEG DRIVEN tDCS}

Further analysis indicated a difference for the bifrontal tDCS group for respectively tinnitus distress $(F=17.72, p<0.001$, $\left.\eta^{2}=0.02\right)$ and tinnitus intensity $\left(F=10.74, p<0.01, \eta^{2}=0.01\right)$. See Figure 2 for an overview.

\section{THE EFFECTS FOR RESPONDERS ONLY}

If we look what patients respond, we found that bifrontal tDCS in comparison to EEG driven tDCS had a larger response rate for both tinnitus distress $\left(\chi^{2}=17.03, p<0.001, \beta=-0.99\right)$ and tinnitus intensity $\left(\chi^{2}=10.41, p<0.01, \beta=-0.68\right)$. That is, for tinnitus distress $19.2 \%$ responded to bifrontal tDCS in comparison to $8.2 \%$ for EEG driven $\mathrm{tDCS}$ and for tinnitus intensity $22.1 \%$ responded to bifrontal tDCS in comparison to $12.5 \%$ for EEG driven $\mathrm{tDCS}$.

A repeated measures ANOVA including only the responders on both distress and intensity revealed a significant main effect for pre versus post measurement $\left(F=115.22, p<0.001, \eta^{2}=0.13\right)$, with a decrease on distress of $36.74 \%$ and on intensity of $28.22 \%$. No significant main effect was obtained for condition (frontal tDCS, and EEG driven tDCS) as well for the interaction effect between condition (frontal tDCS, and EEG driven $\mathrm{tDCS}) \times \mathrm{tDCS}$ (pre versus post) for both.

\section{DISCUSSION}

The main surprising result of the study is that EEG driven placement of anode and cathode does not benefit tinnitus suppression rates in comparison to bifrontal tDCS with anode overlying the right DLPFC and the cathode overlying the left DLPFC. Even though theoretically one would expect that EEG driven tDCS should be superior to "blind" bifrontal stimulation, this does not seem to be case. Multiple explanations can be proposed.

A first explanation is related to the parameter used. Possibly gamma lagged phase synchronization is not a good parameter to determine where to place the cathode. It has indeed been shown, both in the visual system (Antal et al., 2004) and the DLPFC for tinnitus suppression (Vanneste et al., 2011b) that gamma band activity in the area under the cathode is decreased and increased in the area under the anode. However, it is yet unknown whether this also means that gamma functional connectivity as measured by lagged phase synchronization is also modulated. As tDCS brings neurons closer or further away from threshold depending on the polarity, this should not automatically lead to changes in phase synchronization.

A second possible explanation is that even though gamma band activity is important in tinnitus perception, it has also been proposed that this gamma band activity only leads to conscious perception if this activity is connected to a larger network involved in conscious perception (van der Loo et al., 2009; De Ridder et al., 2011a). Gamma band activity, which normally waxes and wanes, and is spatially restricted to small areas, actually is nested on low

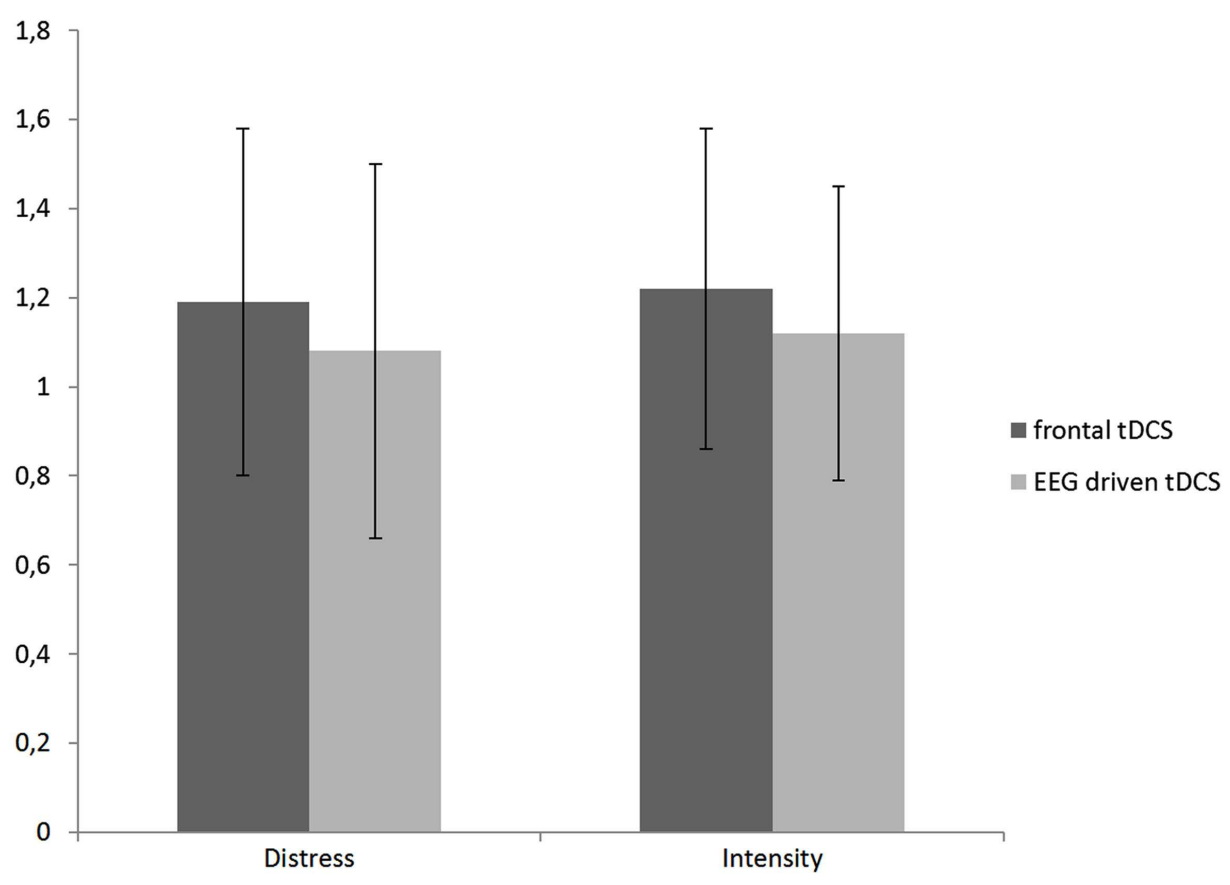

FIGURE 2 |A comparison between the obtained difference (Pre - Post tDCS) for the distress and intensity measurement in respectively frontal and EEG driven tDCS. 
frequency activity, predominantly theta activity, in order to connect to widespread larger networks, both for normal cognition (Canolty et al., 2006; Lisman and Buzsaki, 2008) and in tinnitus (De Ridder et al., 2011a). In a recent study it has been demonstrated that auditory attention control is mediated via gamma band activity in different brain areas, which were connected via theta activity, the phase of which determined gamma synchronization (Doesburg et al., 2012). Thus it could have been better to select theta connectivity as a potential prognostic biomarker, as it is possible theta is a carrier wave on which the information rich gamma activity is nested.

Another explanation can be related to the exactness of the electrode positioning. As gamma band activity is usually spatially restricted and only present in a small focal area, the exact positioning of the cathode and anode might be critically involved in the success of the tDCS stimulation. Since this study was not performed using neuronavigation, because of methodological and technical reasons [(1) sLORETA uses standard head model,
(2) EEG cannot be read into the neuronavigation machine] it cannot be excluded that the electrodes were not spatially correctly positioned (Vanneste et al., 2011b).

Thus, whereas the results of the bifrontal tDCS seem to confirm previous studies (Vanneste et al., 2010c, 2011b; Faber et al., 2011; Frank et al., 2012), in that bifrontal tDCS with anode overlying the right DLPFC and the cathode the left DLPFC has a beneficial effect on tinnitus loudness and distress perception, the use of gamma band functional connectivity seems not bring any advantage to tDCS for tinnitus suppression. Using other potential biomarkers, such as gamma band activity, or theta functional connectivity could theoretically be better alternatives. Further studies will have to elucidate whether brain state based tDCS has any advantages over "blind" bifrontal stimulation.

\section{ACKNOWLEDGMENTS}

The authors thank Jan Ost, Bram Van Achteren, Bjorn De Vree, and Pieter Van Looy for their help in preparing this manuscript.

\section{REFERENCES}

Alain, C., Woods, D. L., and Knight, R. T. (1998). A distributed cortical network for auditory sensory memory in humans. Brain Res. 812, 23-37.

Antal, A., Nitsche, M. A., Kincses, T. Z., Lampe, C., and Paulus, W. (2004). No correlation between moving phosphene and motor thresholds: a transcranial magnetic stimulation study. Neuroreport 15, 297-302.

Axelsson, A., and Ringdahl, A. (1989). Tinnitus - a study of its prevalence and characteristics. Br. J. Audiol. 23, 53-62.

Baguley, D. M. (2002). Mechanisms of tinnitus. Br. Med. Bull. 63, 195-212.

Bodner, M., Kroger, J., and Fuster, J. M. (1996). Auditory memory cells in dorsolateral prefrontal cortex. $\mathrm{Neu}$ roreport 7, 1905-1908.

Canolty, R. T., Edwards, E., Dalal, S. S., Soltani, M., Nagarajan, S. S., Kirsch, H. E., Berger, M. S., Barbaro, N. M., and Knight, R. T. (2006). High gamma power is phase-locked to theta oscillations in human neocortex. Science 313, 1626-1628.

Congedo, M. (2002). EureKa! (Version 3.0) [Computer Software]. Knoxville: NovaTech EEG Inc.

De Ridder, D., De Mulder, G., Verstraeten, E., Seidman, M., Elisevich, K., Sunaert, S., Kovacs, S., van der Kelen, K., van de Heyning, P., and Moller, A. (2007). Auditory cortex stimulation for tinnitus. Acta Neurochir. Suppl. 97(Pt 2), 451-462.

De Ridder, D., Elgoyhen, A. B., Romo, R., and Langguth, B. (2011a). Phantom percepts: tinnitus and pain as persisting aversive memory networks. Proc. Natl. Acad. Sci. U.S.A. 108, 8075-8080.
De Ridder, D., Vanneste, S., and Congedo, M. (2011b). The distressed brain: a group blind source separation analysis on tinnitus. PLOS ONE 6, e24273. doi:10.1371/journal.pone.0024273

De Ridder, D., van der Loo, E., Vanneste, S., Gais, S., Plazier, M., Kovacs, S., Sunaert, S., Menovsky, T., and van de Heyning, P. (2011c). Theta-gamma dysrhythmia and auditory phantom perception. J. Neurosurg. 114, 912-921.

Doesburg, S. M., Green, J. J., McDonald, J. J., and Ward, L. M. (2012). Theta modulation of interregional gamma synchronization during auditory attention control. Brain Res. 1431, 77-85.

Dymond, A. M., Coger, R. W., and Serafetinides, E. A. (1975). Intracerebral current levels in man during electrosleep therapy. Biol. Psychiatry 10, 101-104.

Eggermont, J. J., and Roberts, L. E. (2004). The neuroscience of tinnitus. Trends Neurosci. 27, 676-682.

Faber, M., Vanneste, S., Fregni, F., and De Ridder, D. (2011). Top down prefrontal affective modulation of tinnitus with multiple sessions of tDCS of dorsolateral prefrontal cortex. Brain Stimul. doi: 10.1016/j.brs.2011.09.003

Frank, E., Schecklmann, M., Landgrebe, M., Burger, J., Kreuzer, P., Poeppl, T. B., Kleinjung, T., Hajak, G., and Langguth, B. (2012). Treatment of chronic tinnitus with repeated sessions of prefrontal transcranial direct current stimulation: outcomes from an open-label pilot study. J. Neurol. 259, 327-333.

Fregni, F., Marcondes, R., Boggio, P. S., Marcolin, M. A., Rigonatti, S.
P., Sanchez, T. G., Nitsche, M. A., and Pascual-Leone, A. (2006). Transient tinnitus suppression induced by repetitive transcranial magnetic stimulation and transcranial direct current stimulation. Eur. J. Neurol. 13, 996-1001

Garin, P., Gilain, C., Van Damme, J. P., de Fays, K., Jamart, J., Ossemann, M., and Vandermeeren, Y. (2011). Short- and long-lasting tinnitus relief induced by transcranial direct current stimulation. J. Neurol. 258, 1940-1948.

Heller, A. J. (2003). Classification and epidemiology of tinnitus. Otolaryngol. Clin. North Am. 36, 239-248.

Jastreboff, P. J. (1990). Phantom auditory perception (tinnitus): mechanisms of generation and perception. Neurosci. Res. 8, 221-254.

Knight, R. T., Scabini, D., and Woods, D. L. (1989). Prefrontal cortex gating of auditory transmission in humans. Brain Res. 504, 338-342.

Langguth, B., Eichhammer, P., Kreutzer, A., Maenner, P., Marienhagen, J., Kleinjung, T., Sand, P., and Hajak, G. (2006). The impact of auditory cortex activity on characterizing and treating patients with chronic tinnitus - first results from a PET study. Acta Otolaryngol. Suppl. 556, 84-88.

Leaver, A. M., Renier, L., Chevillet, M. A., Morgan, S., Kim, H. J., and Rauschecker, J. P. (2011). Dysregulation of limbic and auditory networks in tinnitus. Neuron 69, 33-43.

Lewis, J. W., Beauchamp, M. S., and DeYoe, E. A. (2000). A comparison of visual and auditory motion processing in human cerebral cortex. Cereb. Cortex 10, 873-888.

Lisman, J., and Buzsaki, G. (2008). A neural coding scheme formed by the combined function of gamma and theta oscillations. Schizophr. Bull.34, 974-980.

Lockwood, A. H., Salvi, R. J., Burkard, R. F., Galantowicz, P. J., Coad M. L., and Wack, D. S. (1999). Neuroanatomy of tinnitus. Scand. Audiol. Suppl. 51, 47-52.

Lorenz, I., Muller, N., Schlee, W., Hartmann, T., and Weisz, N. (2009). Loss of alpha power is related to increased gamma synchronization-A marker of reduced inhibition in tinnitus? Neurosci. Lett. 453, 225-228.

Miranda, P. C., Lomarev, M., and Hallett, M. (2006). Modeling the current distribution during transcranial direct current stimulation. Clin. Neurophysiol. 117, 1623-1629.

Mitchell, T. V., Morey, R. A., Inan, S., and Belger, A. (2005). Functional magnetic resonance imaging measure of automatic and controlled auditory processing. Neuroreport 16, 457-461.

Muhlau, M., Rauschecker, J. P., Oestreicher, E., Gaser, C., Rottinger, M., Wohlschlager, A. M., Simon, F., Etgen, T., Conrad, B., and Sander, D. (2006). Structural brain changes in tinnitus. Cereb. Cortex 16, 1283-1288.

Muhlnickel, W., Elbert, T., Taub, E. and Flor, H. (1998). Reorganization of auditory cortex in tinnitus. Proc. Natl. Acad. Sci. U.S.A. 95, 10340-10343.

Nitsche, M. A., Fricke, K., Henschke, U., Schlitterlau, A., Liebetanz, D., Lang, N., Henning, S., Tergau, F., and Paulus, W. (2003). Pharmacological modulation of cortical excitability shifts induced by transcranial direct current stimulation in humans. $J$. Physiol. (Lond.) 553(Pt 1), 293-301. 
Nitsche, M. A., and Paulus, W. (2000). Excitability changes induced in the human motor cortex by weak transcranial direct current stimulation. J. Physiol. (Lond.) 527( $\mathrm{Pt} \mathrm{3),}$ 633-639.

Nitsche, M. A., and Paulus, W. (2001). Sustained excitability elevations induced by transcranial DC motor cortex stimulation in humans. Neurology 57, 1899-1901.

Norena, A., Cransac, H., and CheryCroze, S. (1999). Towards an objectification by classification of tinnitus. Clin. Neurophysiol. 110, 666-675.

Pascual-Marqui, R. (2007a). Instantaneous and Lagged Measurements of Linear and Nonlinear Dependence Between Groups of Multivariate Time Series: Frequency Decomposition. Available at: http://arxiv.org/abs/0711.1455

Pascual-Marqui, R. (2007b). Discrete, 3D Distributed, Linear Imaging Methods of Electric Neuronal Activity. Part 1: Exact, Zero Error Localization. Available at: http://arxiv.org/abs/0710.3341

Plewnia, C., Reimold, M., Najib, A., Brehm, B., Reischl, G., Plontke, S. K., and Gerloff, C. (2007). Dosedependent attenuation of auditory phantom perception (tinnitus) by PET-guided repetitive transcranial magnetic stimulation. Hum. Brain Mapp. 28, 238-246.

Rauschecker, J. P., leaver, A. M., and Muhlau, M. (2010). Tuning out the noise: limbic-auditory interactions in tinnitus. Neuron 66, 819-826.

Rush, S., and Driscoll, D. A. (1968). Current distribution in the brain from surface electrodes. Anesth. Analg. 47, 717-723.

Schlee, W., Mueller, N., Hartmann, T., Keil, J., Lorenz, I., and Weisz, N. (2009a). Mapping cortical hubs in tinnitus. BMC Biol. 7, 80. doi:10.1186/1741-7007-7-80

Schlee, W., Hartmann, T., Langguth, B., and Weisz, N. (2009b). Abnormal resting-state cortical coupling in chronic tinnitus. BMC Neurosci. 10, 11. doi:10.1186/1471-2202-10-11

Smits, M., Kovacs, S., de Ridder, D., Peeters, R. R., van Hecke, P., and Sunaert, S. (2007). Lateralization of functional magnetic resonance imaging (fMRI) activation in the auditory pathway of patients with lateralized tinnitus. Neuroradiology 49, 669-679.

van der Loo, E., Congedo, M., Vanneste, S., De Heyning, P. V., and De Ridder, D. (2011). Insular lateralization in tinnitus distress. Auton. Neurosci. doi: 10.1016/j.autneu.2011. 06.007

van der Loo, E., Gais, S., Congedo, M., Vanneste, S., Plazier, M., Menovsky, T., Van de Heyning, P., and De Ridder, D. (2009). Tinnitus intensity dependent gamma oscillations of the contralateral auditory cortex. PLoS ONE 4, e7396. doi:10.1371/journal.pone.0007396

Vanneste, S., de Heyning, P. V., and Ridder, D. D. (2011a). Contralateral parahippocampal gamma-band activity determines noise-like tinnitus laterality: a region of interest analysis. Neuroscience 29, 481-490.

Vanneste, S., Focquaert, F., van de Heyning, P., and De Ridder, D. (2011b). Different resting state brain activity and functional connectivity in patients who respond and not respond to bifrontal tDCS for tinnitus suppression. Exp. Brain Res. 210 217-227.

Vanneste, S., van de Heyning, P., and De Ridder, D. (2011c). The neural network of phantom sound changes over time: a comparison between recent-onset and chronic tinnitus patients. Eur. J. Neurosci. 34 718-731.

Vanneste, S., Plazier, M., van der Loo, E. van de Heyning, P., and De Ridder, D. (2011d). The difference between uni- and bilateral auditory phantom percept. Clin. Neurophysiol. 122, 578-587.

Vanneste, S., and De Ridder, D. (2011). Bifrontal transcranial direct current stimulation modulates tinnitus intensity and tinnitus-distressrelated brain activity. Eur. J. Neurosci. 34, 605-614

Vanneste, S., Plazier, M., der Loo, E., de Heyning, P. V., Congedo, M., and De Ridder, D. (2010a). The neural correlates of tinnitus-related distress. Neuroimage 52, 470-480.

Vanneste, S., Plazier, M., van der Loo, E., Van de Heyning, P., and De Ridder, D. (2010b). The differences in brain activity between narrow band noise and pure tone tinnitus. PLoS ONE 5, e13618. doi:10.1371/journal.pone.0013618

Vanneste, S., Plazier, M., Ost, J., van der Loo, E., Van de Heyning, P., and De Ridder, D. (2010c). Bilateral dorsolateral prefrontal cortex modulation for tinnitus by transcranial direct current stimulation: a preliminary clinical study. Exp. Brain Res. 202, 779-785.

Voisin, J., Bidet-Caulet, A., Bertrand, O., and Fonlupt, P. (2006). Listening in silence activates auditory areas: a functional magnetic resonance imaging study. J. Neurosci. 26 273-278.

Weisz, N., Muller, S., Schlee, W., Dohrmann, K., Hartmann, T., and Elbert, T. (2007). The neural code of auditory phantom perception. $J$. Neurosci. 27, 1479-1484.

Conflict of Interest Statement: The authors declare that the research was conducted in the absence of any commercial or financial relationships that could be construed as a potential conflict of interest.

Received: 01 March 2012; accepted: 04 September 2012; published online: 25 September 2012.

Citation: De Ridder D and Vanneste $S$ (2012) EEG driven $t D C S$ versus bifrontal tDCS for tinnitus. Front. Psychiatry 3:84. doi: 10.3389/fpsyt.2012.00084

This article was submitted to Frontiers in Neuropsychiatric Imaging and Stimulation, a specialty of Frontiers in Psychiatry. Copyright (c) 2012 De Ridder and Vanneste. This is an open-access article distributed under the terms of the Creative Commons Attribution License, which permits use, distribution and reproduction in other forums, provided the original authors and source are credited and subject to any copyright notices concerning any third-party graphics etc. 